\title{
Correlation of Hop test with Speed at thirty meters test in infants between ten and twelve years of a private school in Santiago de Chile \\ Correlación del Single Hop Test con la prueba de Velocidad en treinta metros en infantes entre diez y doce años de un colegio privado de Santiago de Chile \\ Claudio Antonio Oyarzo Mauricio, María Josefina Said Negrete, María Jesús Nazar Araya \\ Univesidad de los Andes (Chile)
}

\begin{abstract}
The speed, fundamental quality in sports, is predicted with horizontal and vertical jumping test. The single hop test, functional evaluation of monopodal long jump has not been studied in this area. Objetive: Correlate hop test with the thirty meters sprint test and with two leg horizontal jump, gold standard in speed prediction. Materials and Method: A study was conducted in pediatric population $(n=91)$ between ten and twelve years of age from a private school in Santiago, Chile. The children were submittted to thirty meters speed test, standing long jump test and hop test with both limbs. The study variables were tested for normal distribution, correlation and linear regression. The same tests were apply per gender and in subjects without asymmetries. Results: The single hop test showed a significant moderate correlation with the time achieved in thirty meters (right $r=-.55$; left $\mathrm{r}=-.58)$ like jump feet together test $(\mathrm{r}=-.49)$. Between jump feet together and the single hop test there was a significant moderate correlation $(\mathrm{r}=.73)$. The single hop test found a 39.566\% of children have one leg jump asymmetry between limbs. There wasn't changes differentiating by gender and by discount the asymmetric subjects. Conclusions: The single hop test is correlate with the sprint in thirty meters similar to the jump feet together and could be used as tool to predict speed in subjects of ten to twelve years. It also detect functional asymmetry of the long jump in children that suggested intervene to prevent possible injury.
\end{abstract}

Keyword. Hop test, thirty meters sprint, speed, functional asymmetry.

Resumen. La velocidad, cualidad fundamental en deportes, se predice con pruebas de salto horizontal y vertical. El single hop test (SHT), evaluación funcional de salto monopodal en longitud, no ha sido estudiado en esta área. Objetivo: Correlacionar single hop test con prueba de velocidad en treinta metros y salto a pies juntos (SPJ), gold estándar en predicción de velocidad. Materiales y Método: Se realizó un estudio en población infantil (n=91) entre diez y doce años de edad de un colegio de Santiago de Chile. Los niños fueron sometidos a pruebas de velocidad a través del tiempo alcanzado en treinta metros, longitud de SPJ y en SHT con ambas extremidades. Las variables en estudio fueron sometidas a pruebas de distribución normal, de correlación y regresión lineal. Las mismas pruebas se aplicaron por sexo y en sujetos sin asimetrías. Resultados: El SHT mostró una correlación significativa moderada con tiempo alcanzado en treinta metros (derecho $r=-55$; izquierdo $r=-.58$ ) al igual que SPJ ( $r=-.49$ ). Entre SHT y SPJ hubo una correlación significativa y moderada ( $\mathrm{r}=$.73). El SHT pesquisó que un 39,5\% de los niños presenta asimetría entre extremidades. No hubo cambios al diferenciar por sexo y al descartar sujetos asimétricos. Conclusiones: El SHT se correlaciona con la velocidad en treinta metros al igual que SPJ y podría ser utilizado como herramienta para predecir velocidad en sujetos de diez a doce años. Además, detecta asimetría funcional del salto en longitud en niños, que se sugiere intervenir para evitar posibles lesiones.

Palabras Clave. Hop test, velocidad en treinta metros, salto a pies juntos, asimetría funcional.

\section{Introducción}

La velocidad destaca entre las cualidades físicas debido a que es un común denominador en el buen desempeño deportivo $(1,2)$. Es muy importante la habilidad de producir una gran fuerza/potencia concéntrica y generar una alta velocidad durante el inicio de la carrera y aceleración (3). Existen numerosas propuestas para mejorar estas dos capacidades $(4,5,6)$. La fuerza y la velocidad son cualidades directamente relacionadas desde el punto de vista fisiológico y están presentes en numerosas pruebas de evaluación de la condición física y el rendimiento deportivo. Habitualmente son medidas a través de la capacidad de salto y la carrera de 30 metros $(7,8,9)$.

Se ha logrado determinar en diversas investigaciones que las pruebas de salto, como manifestación de potencia, son capaces de predecir el desempeño en una prueba de velocidad (2, 7, 10,11, 12). Koklü et al. encontraron una correlación moderada a fuerte entre la velocidad en 30 metros y el salto contramovimiento (2); Maæka ${ }^{3} a$ et al. (3) y Hosseinizarch et al. (11) obtuvieron una correlación significativa entre salto a pies juntos (SPJ) y velocidad en 30 metros; Brechue et al. (10) observaron una alta correlación entre SPJ y velocidad registrada en 9,136.6 y 54.9 metros; Maulder \& Cronin encontraron, para una prueba de 20 metros, una mayor correlación entre velocidad y saltos horizontales, que entre velocidad y saltos verticales (12) y Jiménez-Reyes et al. concluyeron que en el atletismo, el salto de contramovimiento es predictor del rendimiento de competición en las pruebas de velocidad de 100, 200 y 400 metros y en los saltos horizontales (13).

Los test más usados por entrenadores y analistas del movimiento son el salto a pies juntos (SPJ) y el salto vertical (2, 14, 15, 16, 17). La

Fecha recepción: 08-05-16. Fecha de aceptación: 26-12-16 Claudio Antonio Oyarzo Mauricio

coyarzo@miuandes.cl prueba de SPJ es usada como gold standard en la predicción de velocidad en treinta metros y también se utiliza como test de fuerza explosiva de tren inferior, ya que ha demostrado tener buena reproducibilidad y validez con diferentes test de salto vertical (3). Cada una de estas pruebas tiene altos indicadores de confiabilidad, siendo el ICC en SPJ .95 (3), y .97 en la prueba de velocidad en treinta metros (18).

Existe un salto monopodal simple, utilizado en rehabilitación para el reintegro deportivo, denominado single hop test (SHT), que no ha sido correlacionado con la velocidad y que privilegia el salto en longitud, al igual que el SPJ. Esta prueba es utilizada para detectar asimetría funcional entre extremidades $(19,20,21)$, la cual es un reconocido factor de riesgo de lesiones deportivas (22). La utilidad del SHT, la han establecido diversos estudios. Holm, Tveter, Fredriksen \& Vollestad demostraron en una población infantil de siete a doce años, sana y con patología, que es una prueba con mayor sensibilidad y poder descriptivo para evaluar competencia motora que un simple análisis de marcha (23). Fransz, Huurnink, Kingma \& Van Dieën señalan que las pruebas de balance monopodal tiene un lugar importante en la evaluación del desempeño atlético y en el análisis de la evolución en lesiones de extremidad inferior $(21,24)$. Las pruebas que requieren estabilidad postural en mono apoyo, son capaces de reflejar la funcionalidad sensoriomotriz de todo el cuerpo (Witchalls et al., 2013) y aquellas pruebas que demandan habilidades más complejas como los saltos, representan aún mejor la indemnidad de este sistema (Hupperets et al., 2009) por lo que ha aumentado el número de estudios que analizan estas pruebas dinámicas (Fransz et al., 2013). Maulder \& Cronin demostraron que los saltos horizontales son más fiables que los verticales y que un alto número de sujetos tiene desbalance entre extremidades (12). El SHT mide de manera simple, en su desarrollo e implementación, la potencia muscular de manera funcional, el control en la extremidad de prueba y es capaz de detectar asimetría entre extremidades inferiores, (12, 19, 20, 24, 25). Hewit, Cronin \& Hume analizaron diferentes test para detectar asime- 
tría entre extremidades y concluyeron que las mayores asimetrías fueron observadas utilizando el SHT, seguido por el salto lateral y luego el vertical (26). El SHT además cuenta con un índice de confiabilidad alto de .92. $(27,28)$. Johnsen et al. fueron los primeros en concluir la fiabilidad del SHT para evaluar la función de la rodilla en niños activos y deportistas de 12 años promedio (29).

En la literatura se describe el hop index, para determinar una asimetría funcional. Este se expresa en porcentaje y corresponde a la diferencia entre la distancia alcanzada por una extremidad, en relación a la otra. Se ha establecido como norma y referencia clínica, para determinar que un individuo tiene un desempeño normal o simétrico en el SHT, si su rendimiento es e» al 90\% de acuerdo a Daniel o e» al 85\% de acuerdo a Barber. Expresado de otra forma, es considerado un desempeño anormal y asimétrico, si existe una diferencia e» al 10\% o e» al 15\% dependiendo del autor $(28,30)$. Esto ha sido determinado en sujetos con lesión de ligamento cruzado anterior (30) y en sujetos sanos (29, 31).

El objetivo de este estudio fue correlacionar el SHT con la prueba de velocidad en treinta metros y su gold estándar, el SPJ y así proponerla como prueba, no sólo capaz de detectar asimetría entre extremidades en el salto de longitud, sino que también como predictiva de velocidad.

\section{Materiales y Métodos}

\section{Diseño de estudio}

Este estudio fue de tipo observacional y transversal. Se realizaron las mismas pruebas a todos los sujetos. La población no tuvo información previa sobre la realización de la prueba, por lo que no fue una muestra previamente condicionada para lograr un buen desempeño. Los evaluadores, no estaban enterados del desempeño individual de cada niño/a en las pruebas anteriores y ni los niños, ni los profesores, tuvieron la información individual de sus marcas.

\section{Participantes}

En este estudio se utilizó una muestra no aleatoria por conveniencia de ciento once sujetos sanos voluntarios, niños y niñas entre diez y doce años de edad que cursaban cuarto, quinto y sexto básico, en el mismo establecimiento educacional privado de Santiago de Chile, de los cuales noventa y uno fueron los evaluados de forma completa, ya que no tuvieron ausentismo y cumplieron con los criterios de inclusión y exclusión que se registraron a través de un cuestionario. Los criterios de exclusión consideraban la necesidad de no presentar alguna alteración cognitiva, neurológica y/o motora diagnosticada y de alguna lesión de extremidad inferior menor a un año de evolución ya que esto podía limitar el desempeño en las pruebas en estudio. En el colegio evaluado, la totalidad de los alumnos asiste dos a tres veces por semana a clases de actividad física, que involucran actividades deportivas, por lo que se concluye que es una muestra activa. Este estudio fue aprobado por el Comité de Ética de la Universidad de los Andes; Santiago, Chile. Antes de la participación, los padres o apoderados firmaron un consentimiento informado para aprobar la participación de sus pupilos.

\section{Material}

La medición de la estatura y el peso, se realizó con un tallímetro y una balanza electrónica Tanita ${ }^{\circledR}$. Para esto, los niños (as), debían estar con su uniforme de educación física y los pies descalzos. Para las pruebas de salto, se utilizó una cinta métrica y para la prueba de velocidad en treinta metros se utilizó un cronómetro electrónico con programa Sprint Timer de Iphone que incluye fotografía en la meta.

\section{Procedimiento}

Hubo un asentimiento por parte de la totalidad de los alumnos a la participación y se tomaron las medidas de peso y talla.

Previo a las pruebas de velocidad y salto, se realizó un calentamiento de activación utilizado regularmente en las clases escolares de Educación física. Este consistió en; trote de cinco minutos y un calentamiento dinámico no fatigante, realizado en una superficie plana de quince metros de longitud y que contempló una serie de ida y vuelta de: carrera subiendo rodillas buscando longitud en plano sagital, carrera subiendo rodillas buscando altura en plano vertical, carrera lateral, carioca, estocadas y ascensiones (en superficie plana de treinta metros, tres series llegando al ochenta por ciento de velocidad máxima). Luego se realizó la prueba de velocidad en treinta metros en una sola repetición y en series de cuatro alumnos; doce series de varones y luego once series de damas. Terminada la prueba de velocidad, se aplicó la prueba de SHT, primero tres intentos con la extremidad derecha y luego tres intentos con la extremidad izquierda con una pausa de treinta segundos entre saltos. En caso de error en la ejecución, se procedía a repetir el intento. Se eligió el mejor salto de cada extremidad para el análisis estadístico. Finalmente se realizó la prueba de SPJ, con la misma metodología.

No se consideró la familiarización con las pruebas para evitar que la variable fatiga influyera en el rendimiento y de acuerdo a otras investigaciones que utilizaron la misma prueba $(7,20,23,25,28,30,32)$.

El colegio proporcionó listas de alumnos asignadas aleatoriamente. La medición de la longitud de los saltos fue realizada por dos observadores entrenados y el tiempo alcanzado en treinta metros por un juez experimentado en pruebas de campo. Todas las evaluaciones fueron realizadas por los niños con su uniforme de educación física y sus zapatillas personales.

\section{Prueba de velocidad en treinta metros}

Elniño/a en posición de salida desde baja (Figura 1), con la punta del pie avanzado justo tras la línea de salida. Al estímulo auditivo debía correr a máxima velocidad una distancia de treinta metros. Se realizó sólo un intento y fue tomada en pista atlética de ceniza.

\section{Single hop test}

El participante se posicionó en apoyo unipodal con la punta del pie de salto detrás de línea marcada en el piso (Figura 2) y se solicitó un salto horizontal con el objetivo de alcanzar la mayor distancia posible, con las manos tomadas atrás durante toda la ejecución y con la indicación de permanecer en la posición final por al menos dos segundos. La distancia se midió desde la línea de partida, hasta el borde posterior del talón, en la ubicación de la distancia alcanzada.

\section{Salto a pies juntos}

Participante con las manos libres a ambos costados del cuerpo, se posicionó con la punta de ambos pies detrás de la línea marcada en el piso (Figura 3) y saltó en forma horizontal con el objetivo de alcanzar la mayor distancia posible sin impulsarse con los brazos. La distancia se midió desde la línea de partida, hasta el borde posterior del talón más cercano a esta línea, en la ubicación de la distancia alcanzada.

Las mediciones se llevaron a cabo en una superficie lisa de cemento, con una cinta métrica adherida sobre ella, para el registro de la longitud de los saltos.

\section{Análisis estadístico}

Los datos conseguidos del estudio se ingresaron al programa Graph Pad Prism 7.0. Las variables en estudio, tiempo en treinta metros y longitud del salto (single hop test con extremidad derecha SHTD, single hop test de la extremidad izquierda SHTI y salto a pies juntos SPJ), fueron sometidas a tres pruebas para demostrar normalidad en la distribución: Shapiro-Wilk, D-Agostino Pearson y Kolmogorov-Smirnov, las cuales sin excepción demostraron su distribución normal. Luego se determinó la correlación entre el single hop test con extremidad derecha (SHTD), single hop test de la extremidad izquierda (SHTI) y SPJ con la prueba de velocidad en treinta metros. Luego se realizóla misma prueba de correlación, entre el SHT de cada una de las extremidades inferiores con la prueba de SPJ. Posteriormente se llevaron a cabo las mismas correlaciones anteriores separando a la muestra total por sexo y también dejando fuera a los sujetos que presentaron asimetría (una diferencia igual o mayor al 10\% entre extremidades en la prueba de SHT y estos también separados por sexo.

Para todas estas correlaciones se utilizó el coeficiente de correlación de Pearson y se llevaron a cabo análisis de regresión lineal para compro- 


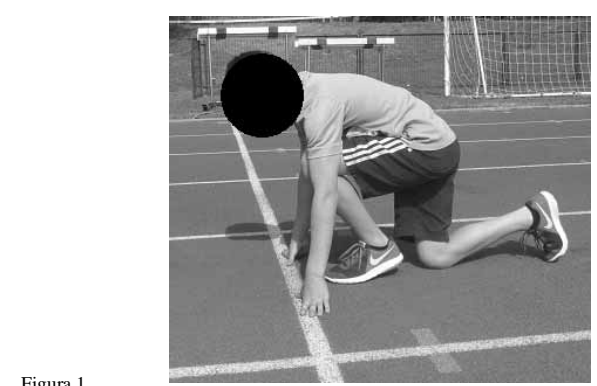

Figura 1.

Salida desde baja, para prueba de velocidad en treinta metros.

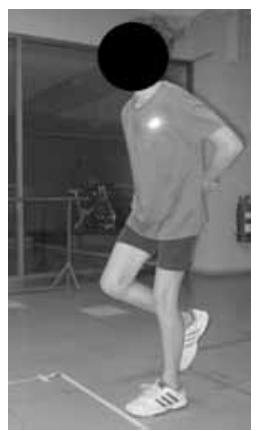

Figura 2

Posición inicial de Hop test. Manos juntas en el dorso bajo con apoyo monopodal.

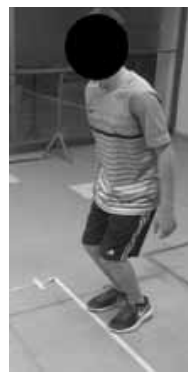

Figura 3.

Posición inicial de prueba Salto a pies juntos. Manos libres al costado del cuerpo con apoyo bipodal.

bar la relación entre las variables. El grado de significación fue establecido en $p>.05$ y con un intervalo de confianza de $95 \%$

\section{Resultados}

Se obtuvo una media de $.94 \pm .19$ metros y $.92 \pm .20$ metros en SHTD y SHTI respectivamente y una media de $1,11 \pm .22$ metros en la distancia alcanzada en la prueba de SPJ y una media de $6.12 \pm .45$ segundos en el tiempo alcanzado en la prueba de velocidad en treinta metros (Tabla 1).

Los resultados de los coeficientes de correlación entre la prueba de velocidad en treinta metros y cada uno de los saltos horizontales, mostraron una correlación significativa $(p>.0001)$ e inversa, es decir, a mayor distancia alcanzada en los saltos, menor fue el tiempo utilizado en lograr los treinta metros (Gráfico 1, 2 y 3).

La correlación entre SHT y velocidad en treinta metros fue moderada. El SHTD tuvo un $r=-.55$, el SHTI un $r=-.58$ y el SPJ un $r=-$ .49 .

Los resultados del coeficiente $\mathrm{r}^{2}$ fueron; SHTD $\mathrm{r}^{2}=.30$, SHTI $\mathrm{r}^{2}=$ .33 y SPJ $\mathrm{r}^{2}=.24$, indicando que alrededor de un 30\% del tiempo

Tabla 1.

\begin{tabular}{|c|c|c|c|c|c|c|}
\hline & \multicolumn{2}{|c|}{ Tiempo $30 \mathrm{~m}(\mathrm{~s})$} & SHTD (m) & \multicolumn{2}{|l|}{ SHTI (m) } & SPJ (m) \\
\hline Media & \multirow{2}{*}{\multicolumn{2}{|c|}{$\begin{array}{l}6.12 \\
.45\end{array}$}} & .94 & \multirow{2}{*}{\multicolumn{2}{|c|}{.92}} & 1.11 \\
\hline $\mathrm{DE}$ & & & .19 & & & .22 \\
\hline \multicolumn{7}{|c|}{$\begin{array}{l}\text { SHTD: single hop test con extremidad derecha, SHTI: single hop test con extremidad } \\
\text { izquierda, SPJ: salto a pies juntos. }\end{array}$} \\
\hline \multicolumn{7}{|c|}{$\begin{array}{l}\text { Tabla } 2 . \\
\text { Niños/as simétricos y asimétricos. }\end{array}$} \\
\hline & Niños & $\begin{array}{c}\text { Porcentaje } \\
\text { masculino (\%) }\end{array}$ & Niñas & $\begin{array}{c}\text { Porcentaje } \\
\text { femenino (\%) }\end{array}$ & Total & $\begin{array}{c}\text { Porcentaje } \\
\text { total (\%) }\end{array}$ \\
\hline Simétricos & 26 & 53.06 & 29 & 69.05 & 55 & 60.44 \\
\hline Asimét & 23 & 46.94 & 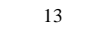 & 30.95 & 36 & 39.56 \\
\hline Total & 49 & 100 & 42 & 100 & 91 & 100 \\
\hline
\end{tabular}

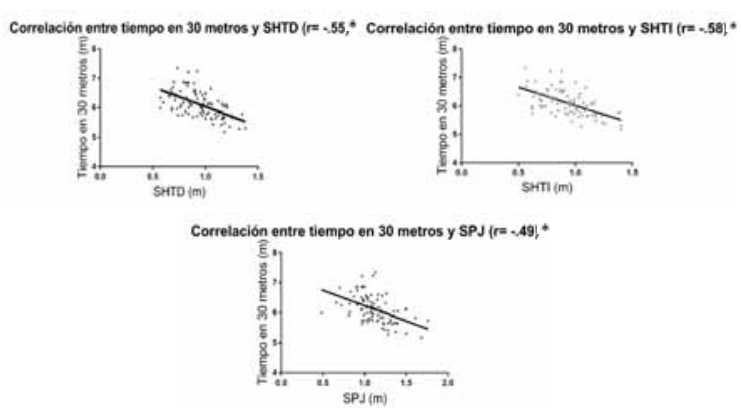

Gráfico 1,2 y 3.

Regresión lineal para relacionar el tiempo de velocidad en treinta metros y el SHTD, SHTI y SPJ respectivamente en la totalidad de la muestra.

SHTD: single hop test con extremidad derecha, SHTI: single hop test con extremidad izquierda, SPJ: salto a pies juntos. * : Indica correlación significativa.

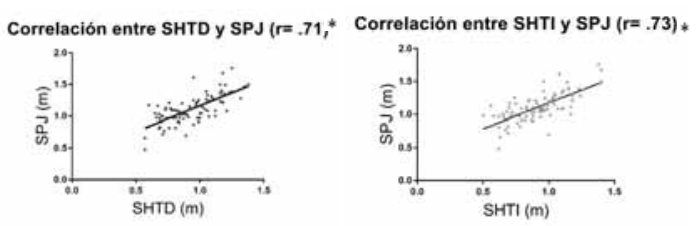

Gráfico 4 y 5.

Regresión lineal entre SPJ y el SHTD Y SHTI.

SHTD: single hop test con extremidad derecha, SHTI: single hop test con extremidad izquierda, SPJ: salto a pies juntos. * : Indica correlación significativa.

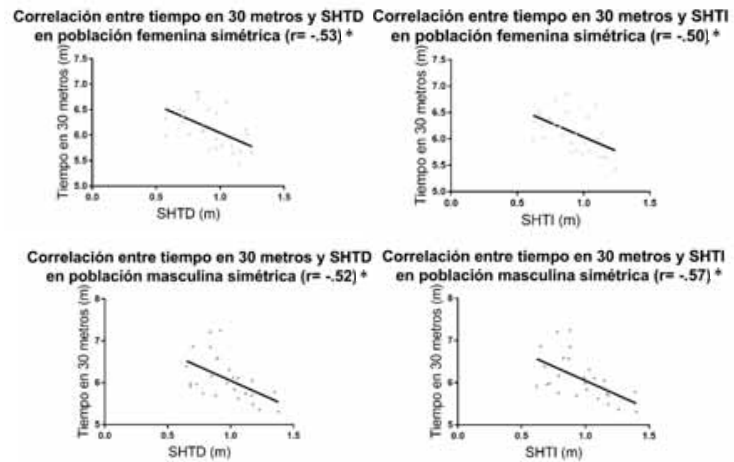

Gráficos 6, 7, 8 y 9 .

Regresión lineal entre tiempo de velocidad en treinta metros y el SHTD Y SHTI en población femenina y masculina simétrica.

SHTD: single hop test con extremidad derecha, SHTI: single hop test con extremidad izquierda. *: Indica correlación significativa.

obtenido en la velocidad en treinta metros se debe a la distancia alcanzada en el SHTD, un 33\% al SHTI y un 24\% al SPJ.

En el análisis de la presencia de asimetría en los niños, se obtuvo en treinta y seis casos una asimetría entre extremidades inferiores igual o mayor al $10 \%$, correspondientes al 39.56\% de la muestra evaluada. Los niños obtuvieron un mayor porcentaje de asimetría (46.94\%) que las niñas (30,95\%) (Tabla 2).

Al correlacionar nuevamente los datos, sin los niños con asimetría igual o mayor al $10 \%$ entre extremidades, se siguió observando una correlación significativa ( $p>$.0001) y un moderado nivel de correlación; SHTD $r=-.53$, el SHTI $r=-.54$, y el SPJ $r=-.46$.

Los valores del coeficiente $\mathrm{r}^{2}$ fueron: SHTD .28, el SHTI .29 y el SPJ .21.

Correlacionando el SHT con la prueba de SPJ, se obtuvo una correlación significativa con un $p<.0001$ y con un valor r moderado de .71 en SHTD y .73 en SHTI (Gráfico 4 y 5).

$\mathrm{Al}$ realizar las pruebas estadísticas por sexo, también se obtienen datos significativos $(p<.0001)$. Ambos obtienen un r moderado; niñas: SHTD r $=-.58$, SHTI $r=-.62$ y niños: SHTD $r=-.53$, SHTI $r=-.54$.

$\mathrm{Al}$ realizar las pruebas por sexo en sujetos simétricos, tanto la población femenina como masculina vuelven a obtener correlaciones significativas y con un r moderado. (Gráfico 6, 7, 8 y 9). 


\section{Discusión}

Los resultados de nuestro estudio muestran una correlación significativa y moderada entre el SHT y la prueba de velocidad en treinta metros con un nivel de correlación similar e incluso mayor que la obtenida por SPJ. En otros estudios, el SPJ ha demostrado una correlación mayor con el tiempo en 30 metros, con valores de $\mathrm{r}=-.62 \mathrm{a}-.80(3,10)$. Esto podría estar dado por la edad de la muestra, ya que en estas investigaciones los sujetos eran atletas y jugadores masculinos de futbol americano con una edad promedio de 21 años y se conoce que ocurre un incremento lineal de la fuerza muscular con la edad durante la infancia (9, 22, 23, 33), lo que les otorga un potencial mayor desarrollo de la fuerza, potencia, balance y coordinación y una mayor experiencia deportiva en los saltos. Sin embargo, Mackala et al. (3) no encontraron diferencias significativas entre atletas y estudiantes en la correlación entre SPJ y tiempo en 30 metros (-.66). Hosseinizarch et al. utilizaron una muestra escolar igual en edad a la de este estudio, pero sólo de sexo femenino y la prueba de velocidad utilizada fue de 45 metros. Encontraron una correlación de -.54 con el SPJ en niñas de 12 años y aún mayor en niñas de 10 y 11 años: -.71 y -.74 respectivamente, siendo una media de -.66 (11).El movimiento horizontal es un componente fundamental en muchos deportes (28) y una característica del SHT y SPJ es su predominio en longitud horizontal, sin embargo, utilizan fuerza de propulsión horizontal y vertical (11), lo que es importante ya que la carrera tiene ambos componentes (28). Maulder y Cronin, demostraron que los saltos horizontales son mejores predictores de velocidad que los verticales, con una correlación de r $=-.73$ a -.86 y r=-.52 a -.73 respectivamente. Dentro de los saltos horizontales, los saltos cíclicos como el salto horizontal repetitivo (tres saltos máximos con una extremidad y aterrizar en ambas extremidades en el último salto) demostraron tener la mayor correlación con una prueba de carrera en 20 metros (12).

La presente investigación también demostró una correlación significativa y moderada entre el SHT y el SPJ y como esta prueba es considerada gold estándar para predecir el rendimiento del tiempo en treinta metros, por reciprocidad, se refuerza la conclusión de que el SHT también podría ser utilizado como prueba predictiva de velocidad.

Para los autores de este estudio es pertinente evaluar el salto en longitud en forma unipodal ya que puede representar mejor la potencia específica para los patrones de movimiento que se expresan en la carrera ya que la mayoría de las actividades motoras funcionales requieren de propulsión monopodal, tanto en el sentido vertical como horizontal.

El valor $\mathrm{r}^{2}$ se interpreta de manera que ninguno de los saltos evaluados justifica más del $50 \%$ de la variabilidad en el tiempo alcanzado en la prueba de velocidad en treinta metros. Esto podría explicarse porque la carrera, no solo requiere de potencia de extremidad inferior, sino que también requiere de coordinación neuromuscular entre extremidades inferiores y superiores, velocidad de reacción y disponibilidad de sustratos energéticos. Los test utilizados habitualmente y en nuestro estudio son acíclicos, y como la carrera es una actividad cíclica (12), los autores de este estudio, consideran que el triple hop test por su condición cíclica podría lograr una mayor correlación.El análisis de la asimetría funcional en los niños, demostró que en treinta y seis casos existió una asimetría en el salto de longitud entre extremidades inferiores igual o mayor al $10 \%$, que correspondieron al $39.56 \%$ de la muestra evaluada. Holm et al. obtuvieron resultados similares en su estudio, concluyendo que un $42 \%$ mostró tener diferencias mayores al 10\% (23). Al estudiar los datos eliminando los niños con asimetría, se mantuvo la misma correlación entre SHT y tiempo en 30 metros, por lo que la asimetría pareciera no influir en la correlación, aunque no se puede descartar que favorezca una lesión.

Todo lo anterior permite respaldar al SHT como prueba predictiva de velocidad cuya simpleza en su realización y medición, proporciona a profesores de educación física una herramienta fiable y segura para detectar talentos deportivos tempranamente. No se pretende sustituir la prueba de velocidad en 30 metros en la detección de talentos, pero si se sugiere agregar el SHT a la batería de pruebas y especialmente aplicarlo en lugares donde no existen pistas de campo o no se cuenta con las condiciones climáticas adecuadas. A esto se debe sumar la capacidad diferenciadora del SHT para detectar asimetría funcional entre extremidades inferiores. Se podría intervenir a los niños con asimetría para corregirla y aportar en su desarrollo motor. De esta forma se puede aportar en la prevención de lesiones y eventualmente en el rendimiento, si es que se logra demostrar que la corrección de la asimetría puede mejorar la velocidad.

Las limitaciones de este estudio comprenden la ausencia de normalización de los datos con la longitud de pierna de cada sujeto y no considerar la mayor experiencia que podría tener un niño que realiza un deporte extra programático, con la consecuente mayor experticia motriz.

Es preocupante la gran cantidad de asimetrías que se presentan en la población infantil, exponiéndola a un mayor riesgo de lesión. Un próximo desafío es crear un protocolo de entrenamiento que permita corregir el patrón disfuncional.

\section{Conclusiones}

El SHT se correlaciona adecuadamente con la velocidad en treinta metros y con su gold estándar el SPJ, por lo que también podría ser utilizado como predictor de velocidad en niños de diez a doce años.

El SHT es capaz de detectar asimetría funcional entre extremidades en niños entre diez y doce años y dada su alta frecuencia, estimula a la intervención y prevención de lesiones.

\section{Agradecimientos}

Este proyecto fue posible gracias al apoyo de la directiva, departamento de Educación Física, apoderados y alumnos del colegio The Mayflower School, a Carlos Santis, juez de atletismo quien cronometró la prueba de velocidad y al comité de ética de la Universidad de los Andes por la revisión del manuscrito.

\section{Referencias}

(1)Taskin, H. (2008). Evaluating sprinting ability, density of acceleration, and speed dribbling ability of professional soccerplayers with respect to their positions. Journal of Strengh and Conditioning Research, 22(5), 1481-1486.

(2) Köklü, Y., Alemdaroglu, U., Özkan, A., Koz, M. \& Ersöz, G. (2015). The relationship between sprint ability, agility and vertical jump performance in young soccer players. Science \& Sports, 30(1), e1-e5.

(3) Maækaª, K., Fostiak, M. \& Kowalski, K. (2015). Selected Determinants of Acceleration in the $100 \mathrm{~m}$ Sprint. Journal of Human Kinetics, 45: 135-148.

(4) Schultz, F.A., Cavazzoni, P., Carnevale, R.V., Abad, C.C., Kobal, R., Pereira, L.A. \& Loturco, I. (2015). Speed and power predictors of change of direction ability in elite snow athletes. Journal of Human Sport \& Exercise, 10(4): 847-856.

(5) García Asencio, C., Sánchez Moreno, M. \& González Badillo, J.J. (2016). Entrenamiento combinado de fuerza y ejercicios de saltos, efectos sobre el rendimiento en el salto vertical en un grupo de alto nivel de jugadores de voleibol durante una temporada completa de competición. Retos. Nuevas tendencias en educación física, deporte y recreación, 29: 140-143.

(6) Sánchez-Sixto, A. \& Floría, P. (2017). Efecto del entrenamiento combinado de fuerza y pliometría en variables biomecánicas del salto vertical en jugadoras de baloncesto. Retos. Nuevas tendencias en educación física, deportey recreación, 31:114-117.

(7) Fernández García, J. C., Reina Gómez, A., Beas Martínez, M. A. \& Martín Recio, F. J. (2007). Fatiga y rendimiento en la velocidad y salto. International Journal of Medicine and Science of Physical Activity and Sport, 7(26): 99-110.

(8) Rodríguez-Rodríguez, F.J, Curilem Gatica, C., Berral de la Rosa, F.J. \&Almagià Flores, A.A. (2017). Evaluación de la educación física 
escolar en enseñanza secundaria. Retos. Nuevas tendencias en educación física, deporte y recreación, 31: 76-81.

(9) López Gallego, F.J., Lara Sánchez,A.J., Espejo Vacas, N. \& Cachón Zagalaz, J. (2016). Influencia del género, la edad y el nivel de actividad física en la condición física de alumnos de educación primaria. Revisión Bibliográfica. Retos. Nuevas tendencias en educación física, deporte y recreación, 29: 129-133.

(10) Brechue, W.F., Mayhew, J.L. \& Piper, F.C. (2010). Characteristics of sprint performance in college football players. J Strength Cond Res, 24(5): 1169-1178.

(11)Hosseinizarch, S.H. \& Bagherpoor, M. (2014). Can standing long jump predict sprint among children? Intl J Basic Sci Appl Res, 3(2): 130-134.

(12)Maulder, P. \& Cronin, J. (2005). Horizontal and vertical jump assessment: reliability, symmetry, discriminative and predictive ability. Physical Therapy in Sport, 6: 74-82.

(13)Jiménez-Reyes, P. \& Gonzalez-Badillo, J.J. (2011). Control de la carga de entrenamiento a través del CMJ en pruebas de velocidady saltos. Cultura, Ciencia y Deporte, 6(18): 207-217.

(14)Hatze, H. (1998). Validity and reliability of methods for testing vertical jumping performance. Journal of Applied Biomechanics, 14: $127-140$

(15)Ruiz, J.R., Romero, V., Castro, J., Artero, E.G., Ortega, F.B. \& Cuenca, M. (2011). Batería ALPHA-Fitness: test de campo para la evaluación de la condición física relacionada con la salud en niños y adolescentes. Nutr. Hosp., 26(6): 1210-1214.

(16)Torres-Luque, G., Carpio, E., Lara Sanchez,A. \& Zagalaz Sánchez, M.L. (2014). Niveles de condición física de escolares de educación primaria en relación a su nivel de actividad física y al género. Retos. Nuevas tendencias en educación física, deporte y recreación, 25: $17-22$

(17)Romero Boza, S., Feria Madueño, A., Sañudo Corrales, B., De Hoyo Lora, M. \& Del Ojo López, J.J. (2014). Efectos de entrenamiento de fuerza en sistema isoinercial sobre la mejora del CMJ en jóvenes futbolistas de elite. Retos. Nuevas tendencias en educación física, deporte y recreación, 26: 180-182.

(18)Marques, M.C., Izquierdo, M., Ferraz, R., Carneiro, A.L. \& González-Badillo, J.J. (2014). Force plate vertical jump measurements and $30 \mathrm{~m}$ sprint performance in trained athletes: A short report. International SportMed Journal, 15(1): 77-83.

(19)Roos, P.E., Button, K., Sparkes, V. \& Van Deursen, R.W.M. (2014). Altered biomechanical strategies and medio-lateral control of the knee represent incompleterecovery of individuals with injury during single leg hop. Journal of Biomechanics, 47: 675-680.

(20)D’Alessandro, R.L., Paolinelli, E.A., Saldanha dos Anjos, M.T., Da Silva, A.A. \& Teixeira da Fonseca, S. (2005). Analysis on the association between isokinetic dynamometry of the knee's articulation and one-leg horizontal jump, hop test, in volleyball athletes. Rev Bras,Med Esporte, 11(5): 255-258.

(21)Christino, M.A., Fleming, B.C., Machan, J.T. \& Shalvoy, R.M. (2016). Psychological factors associated with anterior cruciate ligament reconstruction recovery. Orthop J Sports Med, 4(3): 1-9.

(22)Seger, J.Y. \& Thorstensson, A. (2000). Muscle strength and electromyogram in boys and girls followed through puberty. Eur $J$ Appl Physiol, 81: 54-61.

(23)Holm, I., Tveter, A.T., Fredriksen, P.M. \& Vollestad, N. (2009). A normative sample of gait and hopping on one leg parameters in children 7-12 years of age. Gait \& Posture, 29: 317-321.

(24)Fransz, D.P., Huurnink, A., Kingma, I. \& Van Dieën, J.H. (2014). How does postural stability following a single leg drop jump landin g task relate to postural stability during a single les stance balance task? Journal of Biomechanics, 47:3248-3253.

(25)Zouita, A., Moussa, B., Zouita, S., Dziri, C. \& Salah, B. (2009). Single - leg assessment of postural stability and knee functional outcome two years after anterior cruciate ligament reconstruction. Annals of Physical and Rehabilitation Medicine, 52:475-484.

(26)Hewit, J.K., Cronin, J.B. \& Hume, P.A. (2012). Asymmetry in multi- directional jumping tasks. Physical Therapy in Sport, 13: $238-242$.

(27)Reid, A., Birmingham, T., Stratford, P., Alcock, G. \& Giffin, J. (2007). Hop testing provides a reliable and valid outcome measure during rehabilitation after anterior cruciate ligament reconstruction. Physical Therapy, 87(3): 337-349.

(28)Ross, M.D., Langford, B. \& Whelan, P.J. (2002). Test-Retest reliability of 4 single-leg horizontal hop tests. Journal of Strength and Conditioning Researche, 16(4): 617-622.

(29)Johnsen, M.B., Eitzen, I., Moksnes, H. \& Risberg, M.A. (2015). Inter- and intrarater reliability of four single-legged hop tests and isokinetic muscle torquemeasurements in children. Knee Surg Sports Traumatol Arthrosc, 23(7): 1907-1916.

(30)O’Donnell, S., Thomas, S.G. \& Marks, P. (2006). Improving the sensitivity of the hop index in patients with an ACL deficient knee by transforming the hop distance scores. BMC Musculoskeletal Disorders, 7: 9-14

(31)Munro,A.G. \& Herrington, L.C. (2011). Between-session reliability of four hop tests and the agility t-test. Journal of Strength and conditioning Research, 25(5): 1470-1477.

(32)Glaister, M., Howatson, G., Lockey, R.A., Abraham, C.S., Goodwin, J.E. \& McInnes, G. (2007). Familiarization and reliability of multiple sprint running performance índices. Journal of Strenght and Conditioning Research, 21(3): 857-859.

(33)Malina, R.M., Bouchard, C. \& Bar-Or, A. (2014). Growth, maturation and physical activity. Champaign, IL: Human Kinetics.

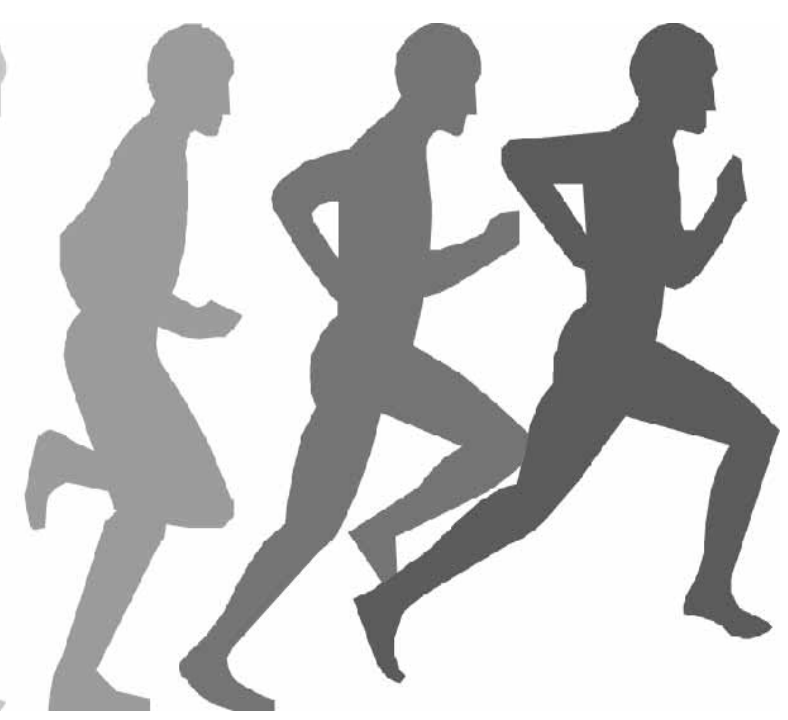

